\title{
Breast density change as a predictive surrogate for response to adjuvant endocrine therapy in hormone receptor positive breast cancer
}

Jisun Kim', Wonshik Han ${ }^{1,2^{*}}$, Hyeong-Gon Moon', Soo Kyung Ahn', Hee-Chul Shin ${ }^{3}$, Jee-Man You, Sae-Won Han, Seock-Ah $\mathrm{Im}^{5}$, Tae-You Kim${ }^{5}$, Hye Ryoung Koo ${ }^{6}$, Jung Min Chang ${ }^{6}$, Nariya Cho ${ }^{6}$, Woo Kyung Moon ${ }^{6}$ and Dong-Young Noh ${ }^{1,2}$

\begin{abstract}
Introduction: Anti-estrogen therapy has been shown to reduce mammographic breast density (MD). We hypothesized that a short-term change in breast density may be a surrogate biomarker predicting response to adjuvant endocrine therapy (ET) in breast cancer.

Methods: We analyzed data for 1,065 estrogen receptor (ER)-positive breast cancer patients who underwent surgery between 2003 and 2006 and received at least 2 years of ET, including tamoxifen and aromatase inhibitors. MD was measured using Cumulus software 4.0 and expressed as a percentage. MD reduction (MDR) was defined as the absolute difference in MD of mammograms taken preoperatively and 8-20 months after the start of ET.

Results: At a median follow-up of 68.8 months, the overall breast cancer recurrence rate was $7.5 \%$ (80/1065). Mean MDR was $5.9 \%$ (range, $-17.2 \%$ to $36.9 \%$ ). Logistic regression analysis showed that age $<50$ years, high preoperative $M D$, and long interval between start of ET to follow-up mammogram were significantly associated with larger MDR $(p<0.05)$. In a survival analysis, tumor size, lymph node positivity, high Ki-67 $(\geq 10 \%)$, and low MDR were independent factors significantly associated with recurrence-free survival $(p<0.05)$. Compared with the group showing the greatest MDR $(\geq 10 \%)$, the hazard ratios for MDRs of $5-10 \%, 0-5 \%$, and $<0 \%$ were $1.33,1.92$, and 2.26 , respectively.
\end{abstract}

Conclusions: MD change during short-term use of adjuvant ET was a significant predictor of long-term recurrence in women with ER-positive breast cancer. Effective treatment strategies are urgently needed in patients with low MDR despite about 1 year of ET.

\section{Introduction}

Adjuvant endocrine therapy is the most effective systemic treatment modality for patients with hormone receptor (ER)-positive breast cancer, although many patients experience tumor recurrence during or after completion of endocrine therapy. Identifying factors that can predict disease recurrence early during adjuvant treatment may result in a more tailored strategy for patients likely to be endocrine resistant and may improve their overall outcomes.

\footnotetext{
* Correspondence: hanw@snu.ac.kr

'Department of Surgery, Seoul National University College of Medicine, 101 Daehakro, Seoul, 110-744, Korea

Full list of author information is available at the end of the article
}

Mammographic breast density (MD) is defined by the relative proportion of radiopaque areas, indicating the presence of fibroglandular tissue among the surrounding fatty component of the breast. High MD is associated with increased risk of breast cancer in both Western and Asian women [1,2]. The degree of lobular involution is known to have inverse correlation with breast cancer risk as well [3].

Studies on the efficacy of tamoxifen for chemoprevention of breast cancer in high-risk women have shown that $\mathrm{MD}$ is decreased following tamoxifen treatment $[4,5]$. Moreover, 12- to 18-month change in MD was found to be an excellent predictor of response to tamoxifen in the preventive setting [5]. However, no studies to date have

\section{Biomed Central}


addressed the association between MD reduction and the efficacy of adjuvant endocrine treatment in breast cancer patients. Using quantitative imaging analysis software to assess serial changes in MD, we investigated the association between the degree of MD reduction and long-term breast cancer recurrence in ER-positive breast cancer patients who received adjuvant endocrine therapy.

\section{Materials and methods}

\section{Study population}

Using our institution's prospectively maintained webbased database, we identified a total of 1,542 ER-positive breast cancer patients who underwent curative surgery at Seoul National University Hospital between October 2003 and December 2006. Patients were excluded if: 1) they did not receive adjuvant endocrine treatment, such as tamoxifen or an aromatase inhibitor, or were treated for less than 2 years; 2) their digital mammogram images were not available; 3) they had bilateral breast cancer, or 4) distant metastasis was observed before the start of endocrine therapy. Clinical and pathologic information on the 1,065 subjects was obtained from the database and used for further analysis. Treatment with adjuvant chemotherapy and/or radiotherapy was generally decided according to the institution's guidelines. The standard duration of treatment with tamoxifen is 5 years. Postmenopausal women were treated with the aromatase inhibitors anastrozole and letrozole for up to 5 years after surgery or after 2 to 3 years of tamoxifen.

\section{Mammographic density measurement}

MD was quantitatively measured on cranio-caudal (CC) images of the unaffected breast using Cumulus software 4.0 (University of Toronto, Toronto, Canada) by a single investigator (JK) blinded to treatment outcome. All evaluated images were digital mammograms performed at our institution, so film scanning was unnecessary. Mammographic density reduction (MDR) was based on two digital mammograms; the first was taken within 2 weeks presurgery (preMD), and the second 8 to 20 months after the start of adjuvant endocrine treatment (postMD), and defined as the absolute difference between the MD of these two images (\% MDR $=\%$ preMD - \% postMD). The MD reduction ratio (MDRR) was also calculated (\% MDRR $=($ preMD - postMD $) \times 100 /$ preMD $)$. Intraobserver reproducibility, tested for $10 \%$ of randomly selected images (213/2,130), was 0.93 (Pearson correlation coefficient).

\section{Statistical analysis}

Change in MD was categorized into four levels, an increase $(\mathrm{MDR}<0), 0 \leq \mathrm{MDR}<5 \%, 5 \leq \mathrm{MDR}<10 \%$, and $\mathrm{MDR} \geq$ $10 \%$, and into a binary variable (MDR $\geq 5 \%$ and $<5 \%$ ), with the $5 \%$ and $10 \%$ absolute reduction cut-offs based on previous findings [5]. We also analyzed absolute MDR as a continuous variable. The chi-square test and $t$-test were used to compare factors that could affect change in MD.

All loco-regional or distant disease recurrences were regarded as recurrence events in recurrence-free survival analysis. Survival curves were estimated by the KaplanMeier method and compared using the log-rank test. Multivariate analyses were conducted using Cox's proportional hazard regression model. All statistical analyses were performed using SPSS (version 17.0) software package (Chicago, IL, USA) and factors with $P<0.05$ were considered statistically significant. Written informed consent was taken prior to surgery in all patients and the study protocol including the use of the database was approved by the Institutional Review Board of Seoul National University Hospital and met the guidelines of the responsible governmental agencies.

\section{Results}

\section{Demographics and result of mammographic density measurement}

The mean age of the 1,065 included patients was 49.1 years (range, 24 to 77 years) (Table 1 ), and their mean duration of overall endocrine therapy was 5.1 years (range, 0.9 to 7.9 years). One hundred and twenty-seven patients (11.9\%) had ductal carcinoma in situ (DCIS). Second mammograms used for density measurements were taken an average 13.1 months (range, 8 to 20 months) after the start of endocrine therapy. The result of MD measurements (preMD, postMD, MDR, and MDRR) are shown in Table 2. Mean MDR was $5.9 \%$ (range, $-17.2 \%$ to $36.9 \%$ ).

\section{Factors associated with density change}

Patients were dichotomized by degree of MD reduction using a cut-off of $5 \%(\mathrm{MDR} \geq 5 \%$ vs. $<5 \%)$, and factors in the two groups were compared to identify associations with high MDR. Patients with $M D R \geq 5 \%$ were significantly younger $(46.5 \pm 8.0$ vs. $51.9 \pm 9.8$ years, $P<0.001$; Additional file 1, Table S1) and were significantly more likely to have been treated with tamoxifen than with an aromatase inhibitor $(P<0.001)$. Similarly, when MDR was analyzed as a continuous variable, mean MDR was higher in patients who received tamoxifen than in those who received an aromatase inhibitor $(6.5 \pm 7.1$ vs. $3.1 \pm 6.3 \%, P$ $<0.001$, data not shown). Mean time from start of endocrine therapy to the second mammogram was longer in the group with $\mathrm{MDR} \geq 5 \%$ than in the group with MDR < $5 \%$ (13.5 \pm 3.1 vs. $12.6 \pm 3.2$ months, $P<0.001)$. Moreover, mean preMD was higher with MDR $\geq 5 \%$ than with MDR $<5 \%(40.9 \pm 12.4 \%$ vs. $30.0 \pm 13.3 \%, P<0.001)$.

Multivariate logistic regression analysis was performed to identify factors independently associated with high MDR (Table 3 ). We found that age $<50$ years, high preoperative MD, and long interval between start of 
Table 1 Patient demographics

\begin{tabular}{|c|c|c|c|}
\hline Variable & Mean \pm SD (range) & Number & $\%$ \\
\hline Age, yr & $49.0 \pm 9.3(24-77)$ & & \\
\hline$\leq 50$ & & 680 & 63.8 \\
\hline$>50$ & & 385 & 36.2 \\
\hline Duration of ET, yr & $5.0 \pm 1.0(0.9-7.9)$ & & \\
\hline \multicolumn{4}{|l|}{ ET regimen } \\
\hline Tamoxifen 5 yr & & 657 & 61.7 \\
\hline Tamoxifen 2-3 yr - > Al (total 5 yr) yr & & 41 & 3.8 \\
\hline Tamoxifen 5 yr - > Al & & 192 & 18 \\
\hline Al $5 \mathrm{yr}$ & & & 16.4 \\
\hline Tumor size $(\mathrm{cm})$ & $2.1 \pm 1.4(0.1-10.0)$ & & \\
\hline$\leq 2 \mathrm{~cm}$ & & 638 & 59.9 \\
\hline$>2 \mathrm{~cm}$ & & 427 & 40.1 \\
\hline \multicolumn{4}{|l|}{ Lymph node status } \\
\hline Negative & & 706 & 66.3 \\
\hline Positive & & 359 & 33.7 \\
\hline \multicolumn{4}{|l|}{ Histologic grade } \\
\hline Low/Intermediate & & 825 & 77.5 \\
\hline High & & 240 & 22.5 \\
\hline \multicolumn{4}{|l|}{ Progesterone receptor } \\
\hline Negative & & 477 & 44.8 \\
\hline Positive & & 588 & 55.2 \\
\hline \multicolumn{4}{|l|}{ HER2 } \\
\hline Negative & & 976 & 91.6 \\
\hline Positive & & 88 & 8.3 \\
\hline \multicolumn{4}{|l|}{ Ki-67 } \\
\hline$<10$ & & 895 & 84 \\
\hline$\geq 10$ & & 169 & 15.9 \\
\hline \multicolumn{4}{|l|}{ Neoadjuvant chemotherapy } \\
\hline No & & 1017 & 95.5 \\
\hline Yes & & 48 & 4.5 \\
\hline \multicolumn{4}{|l|}{ Operation } \\
\hline Breast conserving surgery & & 667 & 62.6 \\
\hline Mastectomy & & 398 & 37.4 \\
\hline \multicolumn{4}{|l|}{ Adjuvant chemotherapy } \\
\hline No & & 247 & 23.2 \\
\hline Yes & & 818 & 76.8 \\
\hline \multicolumn{4}{|l|}{ Radiotherapy } \\
\hline No & & 408 & 38.3 \\
\hline Yes & & 657 & 61.7 \\
\hline \multicolumn{4}{|l|}{ Recurrence } \\
\hline Total & & 80 & 7.5 \\
\hline Locoregional & & 21 & 26.2 \\
\hline Contralateral breast & & 8 & 10 \\
\hline Distant metastasis & & 51 & 63.8 \\
\hline
\end{tabular}

Clinicopathological factors of the 1065 patients. ET: endocrine therapy; Al: aromatase inhibitor; HER2: Human Epidermal Growth Factor Receptor 2.

endocrine therapy and the second mammogram were significantly associated with high MDR $(P<0.05)$. The data were consistently significant on stepwise regression analysis when adjusted by age, preMD and ET regimen (Additional file 1, Table S2).

\section{Density change and recurrence-free survival}

During a median follow-up of 67.7 months, 80 of the 1,065 patients $(7.5 \%)$ experienced tumor recurrence (Table 1). Multivariate Cox regression analysis showed that when analyzed as a continuous variable, MDR was 
Table 2 Distribution of mammographic density before and after treatment and change in mammographic density

\begin{tabular}{|c|c|c|c|}
\hline Variable & Mean (range) & Number & $\%$ \\
\hline PreMD, \%* & $35.77 \pm 13.94(5.42-82.18)$ & & \\
\hline$<10 \%$ & & 26 & 2.4 \\
\hline $10 \%-25 \%$ & & 223 & 20.9 \\
\hline $25 \%-50 \%$ & & 641 & 60.2 \\
\hline$\geq 50 \%$ & & 175 & 16.4 \\
\hline PostMD, \% & $29.84 \pm 12.12(3.90-72.31)$ & & \\
\hline$<10 \%$ & & 35 & 3.3 \\
\hline $10 \%-25 \%$ & & 364 & 34.2 \\
\hline $25 \%-50 \%$ & & 611 & 57.4 \\
\hline$\geq 50 \%$ & & 55 & 5.2 \\
\hline MDR, \% & $5.92 \pm 7.08(-17.2-36.9)$ & & \\
\hline$<5 \%$ & & 505 & 47.4 \\
\hline$\geq 5 \%$ & & 560 & 52.6 \\
\hline$<0 \%$ (increased) & & 190 & 17.8 \\
\hline $0 \%-5 \%$ & & 314 & 29.5 \\
\hline $5 \%-10 \%$ & & 276 & 25.9 \\
\hline$\geq 10 \%$ & & 285 & 26.8 \\
\hline \multicolumn{4}{|l|}{ MDRR, \% } \\
\hline$<15 \%$ & & 486 & 45.6 \\
\hline$\geq 15 \%$ & & 579 & 54.4 \\
\hline$<0 \%$ (increased) & & 190 & 17.8 \\
\hline $0 \%-10 \%$ & & 198 & 18.6 \\
\hline $10 \%-25 \%$ & & 356 & 33.4 \\
\hline$\geq 25 \%$ & & 321 & 30.1 \\
\hline
\end{tabular}

Mammographic density reduction (MDR) and MDR ratio (MDRR) ((preMD postMD) $\times 100 /$ preMD) were initially evaluated as continuous variables then as dichotomized, quartered variables. Patients were divided into two groups (MDR $<5 \%, 5 \% \leq M D R)$, and four groups (MDR $\geq 10 \%, 5 \% \leq M D R<10 \%, 0 \leq$ $M D R<5 \%$, and $M D R<0 \%$ (increased MD)). PreMD: initial preoperative mammographic density; PostMD: density of follow up mammography after 8 to 20 months of hormone therapy.

significantly associated with recurrence-free survival (hazard ratio $(\mathrm{HR})=0.95,95 \%$ confidence interval $(\mathrm{CI})$ 0.92 to $0.99, P=0.005$, Additional file 1 , Table S3). When these patients were categorized into four groups according to the degree of MD change (the reference group with MDR $\geq 10 \%$, plus three groups with MDR of 5 to $10 \%, 0$ to $5 \%$, and < $0 \%$ respectively), the HR for recurrence was proportional to the decrease in MDR.
Compared with the reference group with the greatest MDR reduction ( $\geq 10 \%$ ), the HRs for 5 to $10 \%, 0$ to $5 \%$, and $<0 \%$ MDR were $1.33(P=0.413), 1.92(P=0.048)$, and $2.26(P=0.027)$, respectively (Table 4 , Figure $1 \mathrm{~A})$. Large tumor size, lymph node metastasis, and high Ki67 level ( $\geq 10 \%)$ were also significantly associated with recurrence-free survival. The risk of recurrence was 1.67 times higher with a lower MDR of $<5 \%$ compared to MDR $\geq 5 \%$ when adjusted for age and preMD by forward selection stepwise analysis (HR 1.67, 95\% CI 1.07 to 2.62, $P=0.025$ ) (Additional file 1, Table S4).

HRs for recurrence according to dichotomized MDR $(\geq 5 \%$ vs. $<5 \%)$ in various subgroups are shown in a forest plot (Figure 2). Subgroup analysis showed that the association between low MDR and high risk of recurrence differed by age group (> 50 vs. $\leq 50$ years), with MDR significantly associated with risk of recurrence only in the postmenopausal group. MDR also significantly predicted recurrence in patients taking AIs, and was strongly correlated with the factor of age. When adjusted by age and ET regimen the findings were consistent, showing low MDR as a significant risk factor for recurrence in patients who had undergone chemotherapy (HR $1.70,95 \% \mathrm{CI} 1.04$ to $2.77, P=0.033$ ).

We also calculated the MDRR in this group of patients. We found that MDRR was significantly associated with risk of recurrence when analyzed as a continuous and as a binary variable. Compared with patients with MDRR $\geq 25 \%$, the HRs for recurrence for patients with MDRR of 0 to $10 \%$ and MDRR < $0 \%$ were 2.09 and 2.17 , respectively $(P<0.05$ each; Table 5 , Figure 1B). After adjusting for confounding factors, patients with MDRR $\geq 15 \%$ had a higher risk of recurrence than patients with MDRR < 15\% (HR 1.60, 95\% CI 1.02 to $2.50, P=0.041$ ) (Additional file 1, Table S5).

\section{Discussion}

We have shown here that short-term MDR is predictive of long-term outcomes following endocrine therapy in patients with ER-positive breast cancer. Patients who experienced $<5 \%$ absolute MDR and those with increased MD after about 1 year of endocrine therapy were at 1.92- and 2.26-fold greater risk of recurrence

Table 3 Factors associated with mammographic density reduction (MDR)

\begin{tabular}{lccc}
\hline Variable & Odds ratio & 95\% Confidence interval & $P$-value \\
\hline Age $\leq 50 \mathrm{yr}$ & 1.84 & $1.30,2.61$ & 0.001 \\
Interval to follow-up mammography, months* & 1.07 & $1.02,1.12$ & 0.006 \\
Initial tamoxifen (vs. Al) & 0.99 & $0.65,1.51$ & 0.958 \\
PreMD, \% & 1.06 & $1.04,1.07$ & $<0.001$ \\
Adjuvant chemotherapy & 1.41 & $1.00,2.00$ & 0.052 \\
\hline
\end{tabular}

Factors associated with mammographic density reduction (MDR). Odds ratios are shown for each factor having MDR $<5 \%$ (multivariate logistic regression analysis). *Interval between start of endocrine therapy and follow-up mammography. Al: aromatase inhibitor; PreMD: preoperative mammographic density. 
Table 4 Predictive impact of mammographic density reduction (MDR) on recurrence-free survival

\begin{tabular}{lccc}
\hline Variable & Hazard ratio & 95\% Confidence interval & P-value \\
\hline Age, yr (continuous) & 0.99 & $0.96,1.02$ & 0.393 \\
MDR & & & 0.101 \\
$\geq 10 \%$ (reference) & 1.00 & & 0.413 \\
$5-10 \%$ & 1.33 & $0.67,2.65$ & 0.048 \\
$0-5 \%$ & 1.92 & $1.01,3.64$ & 0.027 \\
$<0 \%$ (increased) & 2.26 & $1.10,4.64$ & 0.006 \\
Size, cm (continuous) & 1.19 & $1.05,1.35$ & 0.008 \\
Lymph node positive & 2.02 & $1.20,3.40$ & 0.323 \\
High histologic grade & 1.29 & $0.78,2.16$ & 0.520 \\
Chemotherapy & 0.79 & $0.39,1.60$ & 0.033 \\
Ki-67 $\geq 10 \%$ & 1.77 & $1.05,3.00$ & \\
\hline
\end{tabular}

Cox proportional hazard regression model for recurrence-free survival. Risk of recurrence was analyzed using the group with the greatest reduction (MDR $\geq 10 \%$ ) as the reference. MDR: absolute mammographic density reduction (pre-treatment - post-treatment).

respectively, than patients with $\mathrm{MDR} \geq 10 \%$. This association was also observed when absolute MDR was analyzed as a continuous variable, and when MDRR rather than absolute MDR was assessed.

Regardless of the evolution of anti-estrogen therapy, a substantial proportion of patients with ER-positive breast cancer experience disease recurrence during follow-up. Effective biomarkers are needed to predict endocrine resistance despite ER expression. Many previous investigations have focused on tumor factors associated with endocrine resistance [6,7]. The level of MDR resulting from endocrine treatment is a host factor indicating individual susceptibility to endocrine agents. These findings support the hypothesis that host response to adjuvant endocrine therapy may be as important and should be considered in addition to the clinicopathological characteristics of the primary tumor.
Breast density is one of the strongest risk factors for breast cancer development [8]. A recent study showed that the magnitude of the association of exogenous or endogenous hormone exposure and mammographic density change is related to future risk of breast cancer [9]. Cuzick et al. conducted a nested case-control study within the IBIS-I study, a randomized prevention trial of tamoxifen versus placebo to determine the association between tamoxifen-induced density change and breast cancer risk. They showed that the 12 - to 18 -month change in mammographic breast density is an excellent predictor of tamoxifen efficacy in the preventive setting [5].

Our findings raise the question of whether treatment strategy should be altered based on change in MD after only one year of endocrine therapy. For the clinical application of individualized therapy, studies are needed
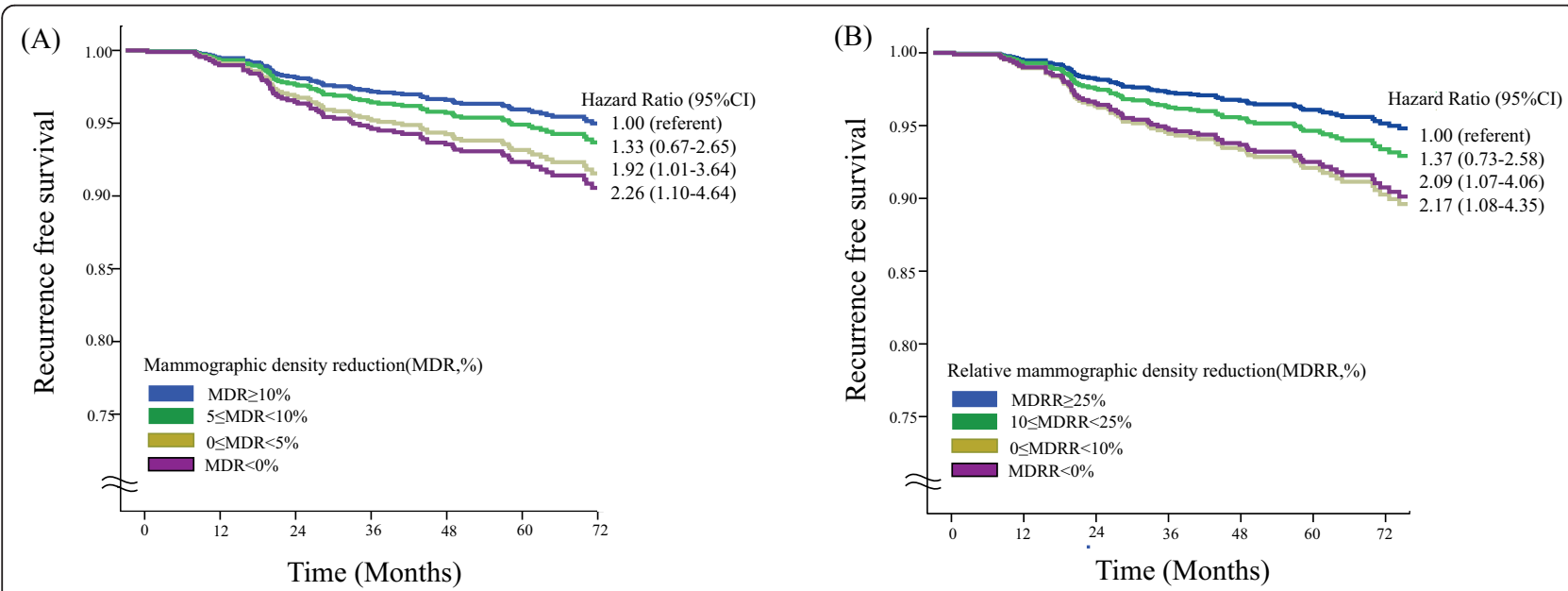

Figure 1 Recurrence-free survival stratified by density reduction group. (A) Recurrence-free survival according to mammographic density reduction (MDR). Patients were divided into four groups, MDR $\geq 10 \%, 5 \% \leq M D R<10 \%, 0 \leq M D R<5 \%$, and MDR $<0 \%$ (increased MD). (B) Recurrence-free survival according to mammographic density reduction ratio (MDRR). Patients were divided into four groups, MDRR $\geq 25 \%, 10 \%$ $\leq$ MDRR $<25 \%, 0 \leq$ MDRR $<10 \%$, and MDRR $<0 \%$ (increased MDRR). Survival curves represent the results of Cox proportional hazard models adjusted for age, tumor size, lymph node, histologic grade, adjuvant chemotherapy, and Ki-67 status. 


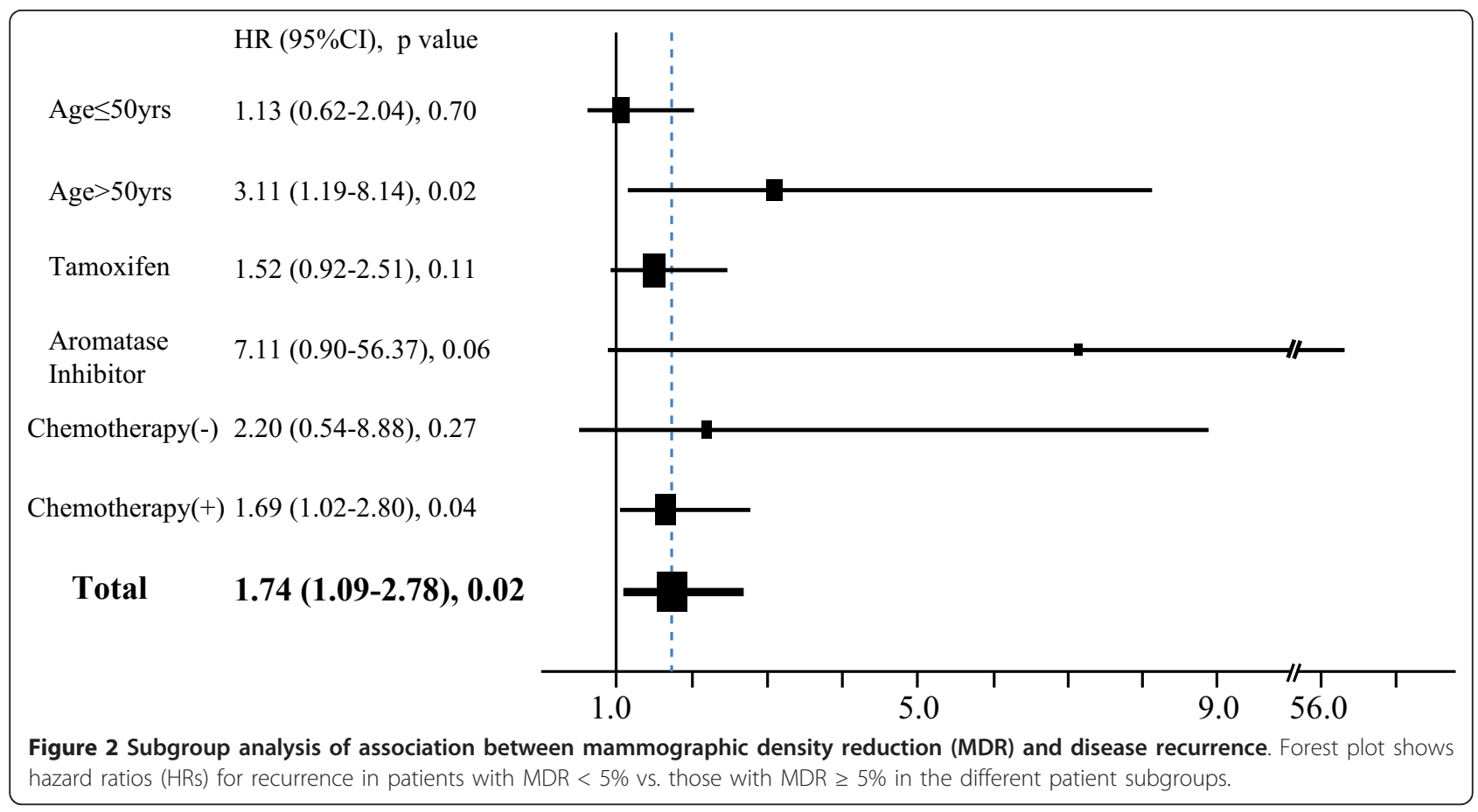

to evaluate the ability of shorter-term changes in MD, such as after $<6$ months, to predict risk of recurrence, and new treatment strategies also should be tested according to the predicted result.

We also found that factors such as age $<50$ years, high preoperative $\mathrm{MD}$, and long interval between the start of endocrine therapy and the second mammogram were significantly associated with high MDR, indicating that a dense breast per se is not a sign of endocrine resistance, and that MD decreases more with prolonged endocrine treatment.

Although MDR was greater in patients $<50$ years of age than in those aged $\geq 50$ years, the degree of MDR was not associated with recurrence in younger patients.
The reason for this is not clear, although it may be due to the complicated hormonal milieu and factors other than endocrine therapy affecting breast density in younger women. The relatively small number of events in patients aged $<50$ years $(43 / 680,7.1 \%)$ in our dataset could have affected the statistical significance and further evaluation within a larger dataset is required.

The degree of MDR may differ according to the type of endocrine therapy. Tamoxifen was reported to be associated with an $8 \%$ mean absolute reduction in breast density at 1.5 years and a reduction of $14 \%$ after 4.5 years $[10,11]$. Raloxifene has been reported to decrease absolute breast density by $1.5 \%$ per year [12]. In a small study involving 54 patients, adjuvant anastrozole had no

Table 5 Predictive impact of mammographic density reduction ratio (MDRR) on recurrence-free survival

\begin{tabular}{cccc}
\hline Variable & Hazard ratio & 95\% Confidence interval & $P$-value \\
\hline Age, yr (continuous) & 0.99 & $0.97-1.02$ & 0.600 \\
MDRR (\%) & & & 0.078 \\
$\geq 25 \%$ & 1.00 & Referent & 0.327 \\
$10 \%-25 \%$ & 1.37 & $0.73-2.58$ & 0.030 \\
$0 \%-10 \%$ & 2.09 & $1.07-4.06$ & 0.030 \\
$<0 \%$ (increased) & 2.17 & $1.08-4.35$ & 0.007 \\
Size, cm (continuous) & 1.19 & $1.05-1.35$ & 0.010 \\
Lymph node positive & 1.98 & $1.18-3.34$ & 0.261 \\
High histologic grade & 1.34 & $0.80-2.24$ & 0.519 \\
Chemotherapy done & 0.79 & $0.39-1.60$ & 0.029 \\
Ki-67 $\geq 10 \%$ & 0.60 & $0.33-0.94$ & \\
\hline
\end{tabular}

Cox proportional hazard regression model for recurrence-free survival. Risk of recurrence was analyzed using the group with the greatest reduction (MDRR $\geq$ $25 \%)$ as the reference. ${ }^{*} \mathrm{MDRR}=($ pre-treatment MD - post-treatment MD) $\times 100 /$ pre-treatment MD. 
effect on breast density in the contralateral breast after 6 months and resulted in a $16 \%$ relative reduction $(P=$ 0.08 ) after 12 months [13]. Letrozole and exemestane also did not reduce mammographic breast density [14]. Addition of an aromatase inhibitor to hormone replacement therapy resulted in a significant reduction in breast density [1]. In a univariate analysis we found that tamoxifen treatment was associated with a higher MDR than treatment with aromatase inhibitors $(P<0.001)$. However, an association between MDR and recurrencefree survival was observed in our aromatase inhibitor group.

There is no definite evidence-based mechanism for an association between anti-estrogen therapy, reduced breast density and a better outcome. With aromatase inhibitors, it is possible that reduced density reflects effective circulating estrogen deprivation, and as a result, also affects micro-metastases. Another explanation is the difference in the drug metabolism efficiency of the host. Patients with adequate serum drug concentrations should have a better response and outcome. However, the metabolic mechanism of tamoxifen and aromatase inhibitors must be different. Adherence to a prescribed drug can partly explain the correlation between density change and patient outcome. In premenopausal and perimenopausal women, a good chemotherapy response could cause chemotherapy-induced ovarian failure, and as a result, could reduce breast density and improve disease-free survival. It is unknown, but is less likely that tumor-associated fibroblasts or other stromal cells in the breast might directly affect distant micro-metastatic cancer cells.

We also found that $17.8 \%$ of our study subjects had increased MD after endocrine therapy. Similarly, in the preventive tamoxifen study IBIS-1, 11\% of patients treated with tamoxifen and $24 \%$ given placebo had an increased MD. The mechanism of this increase in MD is unknown. Investigations are needed to determine whether in these women, tamoxifen acts as an estrogen agonist in the breast.

The reproducibility of MD measurement is important, and studies indicate good intraobserver reproducibility with correlation coefficients of 0.92 to 0.96 [15]. We used Cumulus software, which has been the most widely used for MD measurements in previous studies. Using digital mammographic images, we found that the Pearson correlation coefficient was 0.93 .

The major limitation of the study was the absence of data on factors that may be closely associated with breast density, such as body mass index. Because this study was retrospective in design, the timing of followup mammography was not uniform, varying from 8 to 20 months after initiation of endocrine therapy. Another limitation was that the study subjects received heterogeneous adjuvant therapy regimens. We also could not determine the degree of ER expression in tumors, a potential major factor affecting resistance to endocrine treatment.

To our knowledge, this study was the first to assess the value of MD change as a predictive surrogate in breast cancer patients receiving adjuvant endocrine therapy. The positive result we obtained warrants largerscale prospective studies. Basic research to identify the molecular pathways related to endocrine resistance and mammographic density is also needed.

\section{Conclusion}

In conclusion, low MDR or increased MD during shortterm endocrine therapy was independently associated with poor recurrence-free survival in patients with ERpositive breast cancer. Change in MD may be predictive of response to adjuvant endocrine therapy. Studies should be designed to investigate how to use this valuable information in routine clinical practice.

\section{Additional material}

Additional file 1: Table S1: Univariate analysis for mammographic
density reduction (MDR). Analysis of factors associated with MDR
divided into two group (MDR $<5 \%$ vs. MDR $\geq 5 \%$ ). Younger age,
tamoxifen use, longer interval from initial endocrine therapy, higher
PreMD, adjuvant chemotherapy were likely to have higher MDR ( $\geq 5 \%$ ).
Table S2: Stepwise regression analysis (forward selection) of factors for
MDR* $\geq 5 \%$. After adjusting for the confounding factors, age, interval to
follow up, and preoperative mammographic density, adjuvant
chemotherapy was not independently associated with MDR. Table S3:
Cox proportional analysis for recurrence-free survival (RFS): MDR as a
continuous variable. MDR analyzed as a continuous variable was an
independent risk factor for recurrence, along with size, lymph node (LN)
Status, and Ki-67 level. Table S4: Cox proportional hazard regression
(forward selection) analysis for RFS. After adjusting for confounding
factors, patients with MDR $<5 \%$ had 1.67 times significantly higher risk
of recurrence than the MDR $\geq 5 \%$ group. Tumor size, lymph node (LN)
positivity, and Ki-67 (cut-off $10 \%$ ) were independent prognostic factors as
known. Table S5: Cox proportional hazard regression (forward selection)
analysis for RFS. After adjusting for confounding factors, patients with
MDRR $<15 \%$ had 1.60 times significantly higher risk of recurrence than
the MDRR $\geq 15 \%$ group ( $P=0.041$ ). Tumor size, lymph node (LN)
positivity, and Ki-67 (cut-off $10 \%$ ) were independent prognostic factors as
known.

\section{Abbreviations}

Al: aromatase inhibitor; CC: cranio-caudal; ER: estrogen receptor; DCIS: ductal carcinoma in situ; ET: endocrine therapy; MD: mammographic density; MDR: mammographic density reduction; preMD: initial preoperative mammographic density; postMD: mammographic density after endocrine therapy; MDRR: mammographic density reduction ratio.

\section{Acknowledgements}

This work was supported by a National Research Foundation of Korea (NRF) Grant funded by the Korean Government (20110005753 and 20110031417).

\section{Author details}

${ }^{1}$ Department of Surgery, Seoul National University College of Medicine, 101 Daehakro, Seoul, 110-744, Korea. ${ }^{2}$ Cancer Research Institute, Seoul National 
University, 101 Daehakro, Seoul, 110-744, Korea. ${ }^{3}$ Department of Surgery, Chung-Ang University College of Medicine, 102 Heuksukro, Seoul, 156-755, Korea. ${ }^{4}$ Department of Surgery, Sun General Hospital, 29 Mokjungro, Daejeon, 301-725, Korea. ${ }^{5}$ Department of Internal Medicine, Seoul National University College of Medicine, 101 Daehakro, Seoul, 110-744, Korea.

${ }^{6}$ Department of Radiology, Seoul National University College of Medicine, 101 Daehakro, Seoul, 110-744, Korea.

\section{Authors' contributions}

All the authors have made substantial contributions to conception and design, acquisition of data, or analysis and interpretation of data. HRK, JMC, NC, and WKM carried out analysis of the imaging profiles of each patient including the Cumulus density measurement. SWH, SAI, and TYK confirmed patients' outcomes of recurrence and the adequacy of endocrine therapy. SKA, HCS, and JMY directly participated in the whole process throughout the research and statistical analysis. HGM and DYN participated in the study design and helped to draft the manuscript. JK measured the percent mammographic density and performed the research. As corresponding author, WSH designed and coordinated the research and provided close guidance throughout the process. All authors read and approved the final manuscript. The authors have been involved in drafting the manuscript or revising it critically for important intellectual content and have all given final approval of the version to be published.

\section{Competing interests}

The authors declare that they have no competing interests.

Received: 9 April 2012 Revised: 30 May 2012 Accepted: 6 July 2012 Published: 6 July 2012

\section{References}

1. Mousa NA, Crystal P, Wolfman WL, Bedaiwy MA, Casper RF: Aromatase inhibitors and mammographic breast density in postmenopausal women receiving hormone therapy. Menopause 2008, 15:875-884.

2. Jeon JH, Kang JH, Kim Y, Lee HY, Choi KS, Jun JK, Oh DK, Lee CY, Ko K, Park EC: Reproductive and hormonal factors associated with fatty or dense breast patterns among korean women. Cancer Res Treat 2011, 43:42-48.

3. Ghosh K, Vachon CM, Pankratz VS, Vierkant RA, Anderson SS, Brandt KR, Visscher DW, Reynolds C, Frost MH, Hartmann LC: Independent association of lobular involution and mammographic breast density with breast cancer risk. J Natl Cancer Inst 2010, 102:1716-1723.

4. Boyd NF: Tamoxifen, mammographic density, and breast cancer prevention. J Natl Cancer Inst 2011, 103:704-705.

5. Cuzick J, Warwick J, Pinney E, Duffy SW, Cawthorn S, Howell A, Forbes JF, Warren RM: Tamoxifen-induced reduction in mammographic density and breast cancer risk reduction: a nested case-control study. J Natl Cancer Inst 2011, 103:744-752.

6. Ali S, Coombes RC: Endocrine-responsive breast cancer and strategies for combating resistance. Nat Rev Cancer 2002, 2:101-112.

7. Musgrove EA, Sutherland RL: Biological determinants of endocrine resistance in breast cancer. Nat Rev Cancer 2009, 9:631-643.

8. Boyd NF, Guo H, Martin LJ, Sun L, Stone J, Fishell E, Jong RA, Hislop G, Chiarelli A, Minkin S, Yaffe MJ: Mammographic density and the risk and detection of breast cancer. N Engl J Med 2007, 356:227-236.

9. Boyd NF, Melnichouk O, Martin LJ, Hislop G, Chiarelli AM, Yaffe MJ, Minkin S: Mammographic density, response to hormones, and breast cancer risk. J Clin Oncol 2011, 29:2985-2992.

10. Atkinson C, Warren R, Bingham SA, Day NE: Mammographic patterns as a predictive biomarker of breast cancer risk: effect of tamoxifen. Cancer Epidemiol Biomarkers Prev 1999, 8:863-866.

11. Cuzick J, Warwick J, Pinney E, Warren RM, Duffy SW: Tamoxifen and breast density in women at increased risk of breast cancer. J Natl Cancer Inst 2004, 96:621-628.

12. Freedman M, San Martin J, O'Gorman J, Eckert S, Lippman ME, Lo SC, Walls EL, Zeng J: Digitized mammography: a clinical trial of postmenopausal women randomly assigned to receive raloxifene, estrogen, or placebo. J Natl Cancer Inst 2001, 93:51-56.

13. Prowell TM, Blackford AL, Byrne C, Khouri NF, Dowsett M, Folkerd E, Tarpinian KS, Powers PP, Wright LA, Donehower MG, Jeter SC, Armstrong DK, Emens LA, Fetting JH, Wolff AC, Garrett-Mayer E, Skaar TC,
Davidson NE, Stearns V: Changes in breast density and circulating estrogens in postmenopausal women receiving adjuvant anastrozole. Cancer Prev Res (Phila) 2011, 4:1993-2001.

14. Cigler T, Richardson $H$, Yaffe MJ, Fabian CJ, Johnston D, Ingle JN, Nassif E, Brunner RL, Wood ME, Pater JL, Hu H, Qi S, Tu D, Goss PE: A randomized, placebo-controlled trial (NCIC CTG MAP.2) examining the effects of exemestane on mammographic breast density, bone density, markers of bone metabolism and serum lipid levels in postmenopausal women. Breast Cancer Res Treat 2011, 126:453-461.

15. Byng JW, Boyd NF, Fishell E, Jong RA, Yaffe MJ: The quantitative analysis of mammographic densities. Phys Med Biol 1994, 39:1629-1638.

doi:10.1186/bcr3221

Cite this article as: Kim et al:: Breast density change as a predictive surrogate for response to adjuvant endocrine therapy in hormone receptor positive breast cancer. Breast Cancer Research 2012 14:R102.

\section{Submit your next manuscript to BioMed Central and take full advantage of:}

- Convenient online submission

- Thorough peer review

- No space constraints or color figure charges

- Immediate publication on acceptance

- Inclusion in PubMed, CAS, Scopus and Google Scholar

- Research which is freely available for redistribution 